\title{
Revised Pioneer 10 Absolute Electron Intensities in the Inner Jovian Magnetosphere
}

\author{
D. N. Baker ${ }^{1}$ and J. A. Van Allen \\ Department of Physics and Astronomy, University of Iowa, Iowa City, lowa 52242
}

\begin{abstract}
Using improved techniques for the analysis of the University of lowa Pioneer 10 Jupiter encounter data, we have obtained significantly more reliable values for energetic electron $\left(E_{e}<21 \mathrm{MeV}\right)$ intensities within the inner magnetosphere. Our revised absolute intensities of electrons $0.06<E_{e}<21 \mathrm{MeV}$ are less than our previous estimates by factors as great as 10 for $L \leqslant 6$. Our previously published intensities at greater radial distances for $E_{e}<21 \mathrm{MeV}$ and at all radial distances for $E_{e}>21 \mathrm{MeV}$ are not affected by the revisions.
\end{abstract}

\section{INTRODUCTION}

In a previously published paper concerning our Pioneer 10 experiment [Van Allen et al., 1974] (called paper 1 hereafter), values were given along the Pioneer 10 encounter trajectory for the absolute omnidirectional intensities of Jovian energetic electrons in five integral energy ranges $\left(E_{e}>0.06,0.55,5,21\right.$, and $31 \mathrm{MeV})$. As was made clear, however, the intensities in the three lower-energy ranges in the region interior to the orbit of Europa $(L=9.5)$ were regarded as provisional. The nature of the uncertainties was discussed fully in the section of the paper concerning physical calibration of the instrument. The present paper gives revised absolute intensities for the inner Jovian magnetosphere based on improved data analysis techniques.

The two higher-energy channels of the University of Iowa instrument $\left(E_{\mathrm{e}}>21\right.$ and $\left.31 \mathrm{MeV}\right)$ were relatively free of uncertainties throughout the encounter and are regarded as providing reliable absolute intensities, as published originally. No changes in these values are proposed herein.

The three lower-energy electron intensities were shown as dashed lines in Figure 13 of paper 1 for the inner portion of the encounter. The dashed lines represented the intensities that were inferred formally from the application of nominal geometric factors to our then best estimates of true detector counting rates. Because of instrumental shortcomings at the very high intensities and for the very hard spectra near periapsis this procedure has been found to yield intensities that are too great, especially in the lowest-energy channel. The net effect of the revisions, which will be discussed, is to lower the absolute intensities by factors as great as $\sim 10$ inside Io's orbit. The intensities for $L>9.5$ are essentially unaffected by the revisions and stand as they were originally published.

\section{IMPROVEMENTS IN ANALYSIS}

Paper I gives a full description of our instrument, a discussion of its shortcomings, and our then best estimates of absolute intensities of electrons along the Pioneer 10 trajectory through the magnetosphere of Jupiter. The present short report is an abridged version of University of Iowa Research Report 75-28, available on request [Baker and Van Allen. 1975].

\footnotetext{
${ }^{1}$ Now at George W. Downs Laboratory of Physics, California Institute of Technology, Pasadena, California 91125.
}

Copyright (C) 1977 by the American Geophysical Union.
The principal problem of analysis lies in the characteristic curves of the detectors relating the apparent (observed) counting rate $r$ to the 'true' rate $R$, as would be observed with a detector of zero dead time. (See Figure 8 and the accompanying discussion in paper 1.) Despite comprehensive and careful determination of these characteristic curves in preflight calibration work over an extended period of time, a small upward shift in the value of $r_{\max }$ occurred between launch and encounter with the planet.

We have found it possible to reconstruct the full $r$ versus $R$ curves for detectors $\mathrm{A}, \mathrm{B}$, and $\mathrm{C}$ as they actually were during encounter by using the observed double coincidence rates $r_{\mathrm{AB}}$ and triple coincidence rates $r_{\mathrm{ABc}}$.

Also, we have been able to resolve the basic ambiguity between the left-hand branch and the right-hand branch of the $r$ versus $R$ curves by detailed study of the angular distributions of the apparent counting rates $r$.

Finally, we have found from such angular distributions of the apparent counting rate of the scatter geometry detector $G$ that its response at low $L$ values was dominated by electrons $E_{\mathrm{p}}>1 \mathrm{MeV}$ penetrating a thin portion of its shield and not by electrons $E_{e}>0.060 \mathrm{MeV}$ entering the detector through the collimator, as is the case elsewhere.

\section{RESULTS AND Discussion}

Our final results are summarized in Figure 1 and are shown in more detail in Figure 2. The absolute omnidirectional intensities of electrons are plotted with 3.5-min time resolution, the interval of time required in the data sampling scheme to assemble a full roll angle distribution at the prevailing spacecraft spin period of $12.62 \mathrm{~s}$. As was pointed out in paper 1, there is considerable structure in the intensity time profiles, most particularly near the regions of influence of the Galilean satellites. The geometrical 'sweeping' region associated with each satellite (in a centered dipole field of tilt $9.5^{\circ}$ toward system $3(1957.0)$, longitude $\left.l_{s}=230^{\circ}\right)$ is indicated as a vertical shaded bar in Figures 1 and 2. The detailed structure at the location of a given satellite's orbit is obviously energy dependent as well as dependent on the relative latitudes and longitudes of the spacecraft and the satellite.

Figure 2 shows an enlarged view of the near-periapsis data, including the Europa and Io crossings both inbound and outbound. In addition, we show as dashed lines the provisional intensity estimates of paper 1 . Near closest approach to the planet the intensity of electrons $E_{e}>5 \mathrm{MeV}$ is reduced by a factor of $\sim 3$ from the previous values. The intensity of electrons $E_{e}>0.55 \mathrm{MeV}$ is reduced by a factor of $\sim 3.5$ in the same region. The next to upper curve in Figure 2, derived from the

Paper number 6A0847. 


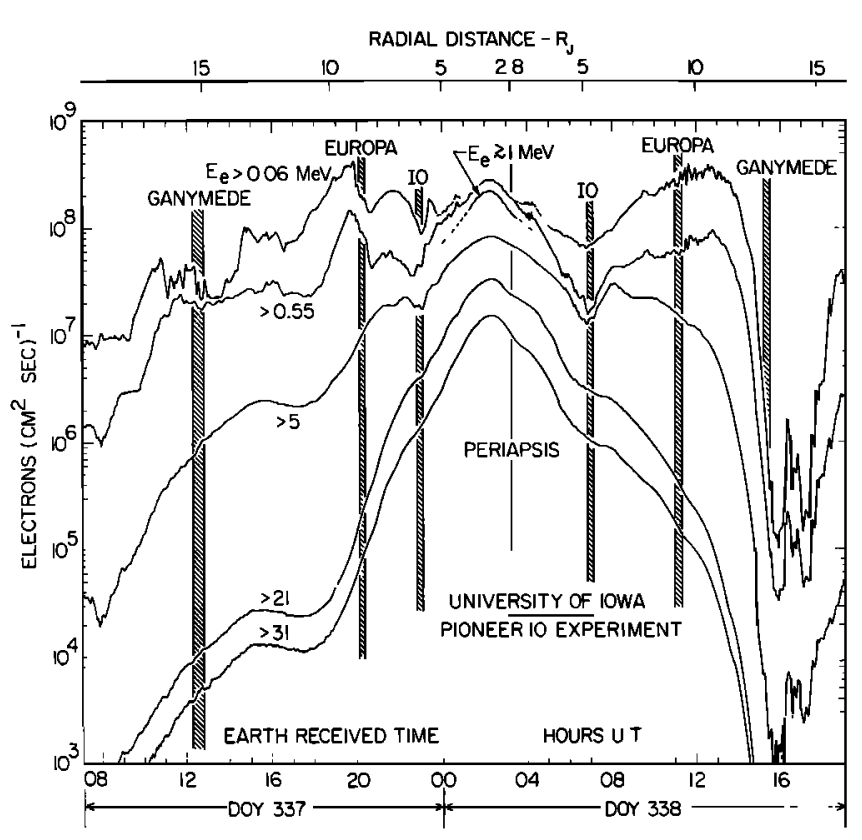

Fig. 1. The absolute omnidirectional intensities of electrons in six integral energy ranges. The intensity of electrons $E_{e} \gtrsim 1 \mathrm{MeV}$ corresponds to particles penetrating the side shielding of the scatter geometry detector $\mathrm{G}$. The geometrical sweeping regions of the several $\mathrm{Gali}$ lean satellites are shown as vertical shaded bars. DOY means day of year 1973.

detector $G$ rates, is broken into two parts. All of the evidence indicates that to well within the Europa $L$ shell the normal scatter counter behavior obtains for $G$. More and more sidewall penetration by higher-energy electrons then begins to take place (as is evidenced by skewing of the angular distribution) until sidewall penetration dominates interior to Io's orbit. Taking these effects into account, we have shown the 0.060 $\mathrm{MeV}$ electron intensity inward in radial distance as far as we can reasonably determine it. An uncertain transition region is shown as a dashed line, and then at lesser radii the $G$ counting rate is reinterpreted to correspond to penetrating electrons $E_{e}$ $>1 \cdot \mathrm{MeV}$. Electron intensities $0.060<E_{\mathrm{e}}<1 \mathrm{MeV}$ in this innermost region cannot exceed $5 \times 10^{8}\left(\mathrm{~cm}^{2} \mathrm{~s}\right)^{-1}$ without violating the observed angular distribution.

The intensity peak just inside the lo $L$ shell on the inbound pass is apparently a reliable feature even though its intensity is poorly determined. The phase angles of the angular distributions in this region are such that the feature is clearly due to low-energy ( $E_{e} \gtrsim 0.060 \mathrm{MeV}$ ) electrons entering the detector via the scatter tube and not penetrating the side shielding. This peak corresponds to a less prominent one in the $E_{e}>0.55$ $\mathrm{MeV}$ profile at a similar location. Fillius and Mcllwain [1974] and $M c l l$ wain and Fillius [1975] have discussed similar features in the University of California at San Diego (UCSD) Pioneer 10 data for electrons $E_{e}>0.16 \mathrm{MeV}$ in terms of injection by the satellite Io as proposed by Gurnett [1972]. Although a small peak occurs in our outbound data for the $0.55-\mathrm{MeV}$ channel, no discernible peak is present for detector G. However, penetrating particles may well be masking the effect in the lowest energy range.

We conclude from Figure 2 that (1) near periapsis the intensities of the low-energy electrons $\left(E_{e} \sim 100 \mathrm{keV}\right)$ are probably lower than the estimates that we gave in paper 1 by a factor as great as 10 and (2) there is an lo-related peak of low- energy electrons at least (and most clearly) for the inbound Pioneer 10 pass.

A significant disagreement existed between the intensities of electrons $E_{e} \sim 100 \mathrm{keV}$ given in paper 1 and those given by Fillius and Mcllwain [1974] for $L \leqq 6$, the latter being about an order of magnitude less than the former. The revised Iowa intensities tend to confirm the lower values of Fillius and Mcllwain inside of Io's orbit. For $L>8$ the original lowa and UCSD intensities in similar energy ranges agreed to within about $50 \%$. In the higher-energy ranges $\left(E_{e}>31\right.$ and $35 \mathrm{MeV}$ for the Iowa and UCSD instruments, respectively) there was no significant disagreement throughout the Pioneer 10 encounter. Our revised data are, however, in significant disagreement (by a factor of $\gtrsim 3$ ) with the higher intensities of electrons $E_{e}>$ $3 \mathrm{MeV}$ reported by the University of Chicago group [Simpson et al., 1974].

Our originally reported electron intensities $E_{e}>21 \mathrm{MeV}$ and $E_{e}>31 \mathrm{MeV}$ seem well determined throughout the encounter and have withstood restudy in the course of our current work.

Finally, it is of interest to mention more recent results from the Pioneer 11 mission on which an improved version of the lowa instrument was carried to within $1.6 R_{J}$ of the center of the planet [Van Allen et al., 1975]. The equatorial plane was crossed at a radius of $1.65 R_{J}$ during the occultation period. At that point the measured intensity of electrons $E_{e}>21 \mathrm{MeV}$ was approximately $1.8 \times 10^{7}\left(\mathrm{~cm}^{2} \mathrm{~s}\right)^{-1}$, i.e., a factor of nearly 2

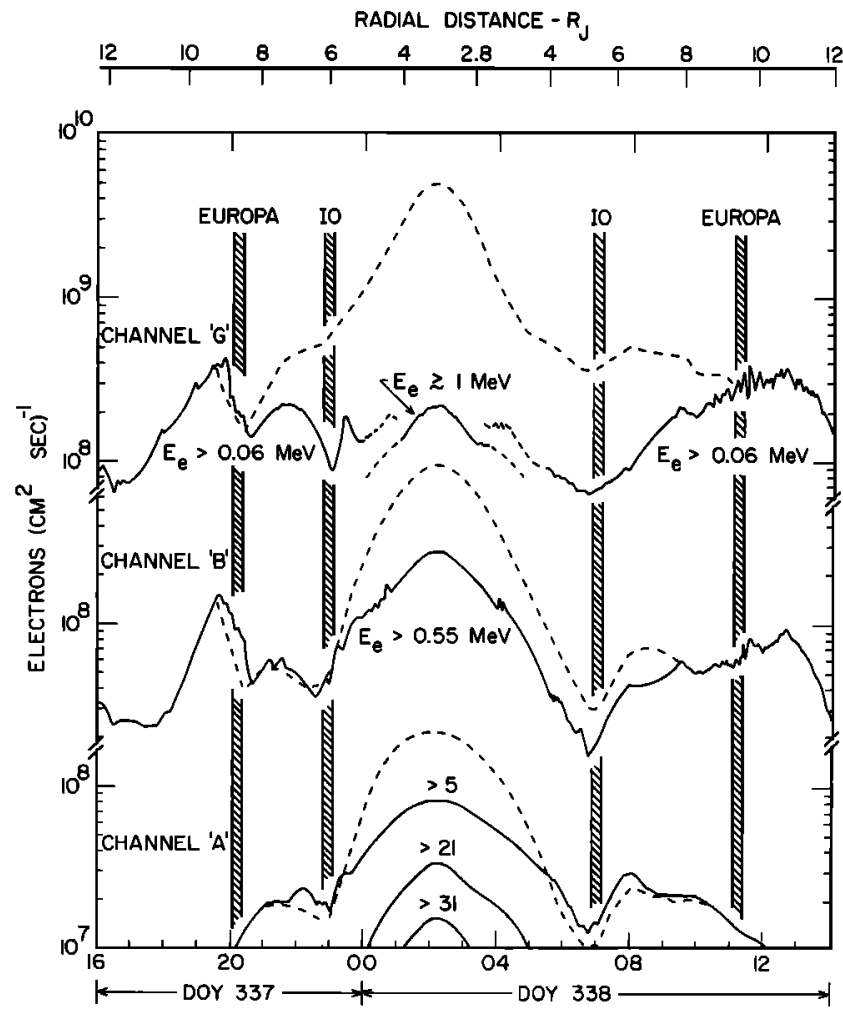

Fig. 2. An enlarged view of the absolute intensity profiles near periapsis including the Europa and Io inbound and outbound $L$ shell crossings. The dashed curves for $E_{e}>0.06,0.55$, and $5 \mathrm{MeV}$ represent the provisional intensity estimates reported in paper 1 . Our revised values are shown as solid lines. Note the two different branches of the detector $G$ curve corresponding to a qualitative difference in its effective response characteristics at large and small radial distances as discussed in the text. 
below the intensity at $3 R_{J}$. The intensities of electrons $E_{e}>$ $0.040 \mathrm{MeV}$ and $E_{\mathrm{e}}>0.56 \mathrm{MeV}$ were nearly equal at the same location, being approximately $6.5 \times 10^{7}\left(\mathrm{~cm}^{2} \mathrm{~s}\right)^{-1}$. Hence the radial profiles for the intensity of lower-energy electrons also 'roll over,' being more than a factor of 4 lower at $1.65 R_{J}$ than at $3 R_{J}$.

The revised Pioneer 10 results and the new Pioneer 11 results are of importance in calculating the expected radio emission at decimetric wavelengths.

Acknowledgments. This work has been supported in large part by Ames Research Center/NASA contract NAS2-6553, by the U.S. Office of Naval Research, and by National Aeronautics and Space Administration research grant NGL 16-001-002. We have received substantial assistance in the reduction and analysis of data from B. A. Randall, D. D. Sentman, and R. L. Brechwald of the Iowa group.

\section{REFERENCES}

Baker, D. N., and J. A. Van Allen, Revised Pioneer 10 absolute electron intensities in the inner Jovian magnetosphere, Res. Rep. 75. 28, Univ. of Iowa, Iowa City, Aug. 1975.
Fillius, R. W., and C. E. Mcllwain, Measurements of the Jovian radiation belts, J. Geophys. Res., 79, 3589-3599, 1974.

Gurnett, D. A., Sheath effects and related charged-particle acceleration by Jupiter's satellite Io, Astrophys. J., 175, 525-533, 1972.

Mcllwain, C. E., and R. W. Fillius, Differential spectra and phase space densities of trapped electrons at Jupiter, J. Geophys. Res. 80. 1341-1345, 1975

Simpson, J. A., D. C. Hamilton, R. B. McKibben, A. Mogro-Campero, K. R. Pyle, and A. J. Tuzzolino, The protons and electrons trapped in the Jovian dipole magnetic field region and their interaction with Io, J. Geophys. Res., 79, 3522-3544, 1974.

Van Allen, J. A., D. N. Baker, B. A. Randall, and D. D. Sentman, The magnetosphere of Jupiter as observed with Pioneer 10,1, Instrument and principal findings, J. Geophys. Res., 79, 3559-3577, 1974.

Van Allen, J. A., B. A. Randall, D. N. Baker, C. K. Goertz. D. D. Sentman, M. F. Thomsen, and H. R. Flindt, Pioneer 11 observations of energetic particles in the Jovian magnetosphere, Science, 188, 459-462, 1975.

(Received August 11, 1975; accepted November 8, 1976.) 